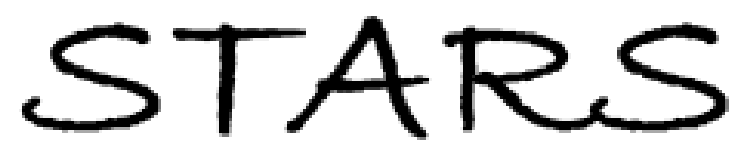

University of Central Florida

STARS

Faculty Bibliography 2000s

Faculty Bibliography

$1-1-2003$

\title{
Prism grating using polymer stabilized nematic liquid crystal
}

Hongwen Ren

University of Central Florida

Yun-Hsing Fan

University of Central Florida

Shin-Tson Wu

University of Central Florida

Find similar works at: https://stars.library.ucf.edu/facultybib2000

University of Central Florida Libraries http://library.ucf.edu

This Article is brought to you for free and open access by the Faculty Bibliography at STARS. It has been accepted for inclusion in Faculty Bibliography 2000 s by an authorized administrator of STARS. For more information, please contact STARS@ucf.edu.

\section{Recommended Citation}

Ren, Hongwen; Fan, Yun-Hsing; and Wu, Shin-Tson, "Prism grating using polymer stabilized nematic liquid crystal" (2003). Faculty Bibliography 2000s. 3982.

https://stars.library.ucf.edu/facultybib2000/3982

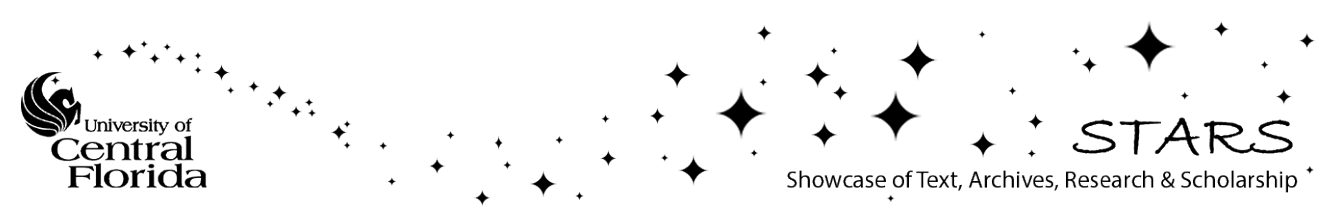




\section{Prism grating using polymer stabilized nematic liquid crystal}

Cite as: Appl. Phys. Lett. 82, 3168 (2003); https://doi.org/10.1063/1.1573366

Submitted: 20 January 2003 . Accepted: 07 March 2003 . Published Online: 06 May 2003

Hongwen Ren, Yun-Hsing Fan, and Shin-Tson Wu

ARTICLES YOU MAY BE INTERESTED IN

Reconfigurable fabrication of scattering-free polymer network liquid crystal prism/grating/ lens

Applied Physics Letters 102, 161106 (2013); https://doi.org/10.1063/1.4802919

Liquid-crystal diffraction gratings using polarization holography alignment techniques

Journal of Applied Physics 98, 123102 (2005); https://doi.org/10.1063/1.2146075

Fast-response and scattering-free polymer network liquid crystals for infrared light modulators

Applied Physics Letters 84, 1233 (2004); https://doi.org/10.1063/1.1649816

\section{Applied Physics Letters}

Mid-IR and THz frequency combs special collection

\section{Read Now!}




\title{
Prism grating using polymer stabilized nematic liquid crystal
}

\author{
Hongwen Ren, Yun-Hsing Fan, and Shin-Tson $\mathrm{Wu}^{\mathrm{a})}$ \\ School of Optics/CREOL, University of Central Florida, Orlando, Florida 32816
}

(Received 20 January 2003; accepted 7 March 2003)

\begin{abstract}
A prism grating using polymer stabilized liquid crystal was fabricated and its diffraction characteristics evaluated. Such a prism grating exhibits not only a periodic grating structure but also a prism-like gradient refractive index within each grating period. As a result, the diffraction behavior is asymmetric. The first order exhibits high diffraction efficiency while the zeroth order has a high extinguishing ratio. The potential application as a beam deflector is emphasized. (c) 2003 American Institute of Physics. [DOI: 10.1063/1.1573366]
\end{abstract}

Various liquid crystal (LC) beam steering devices have been developed for optical interconnects, optical communications, projection displays, and general industrial applications. ${ }^{1-8}$ Among these beam steering devices, the prism type is most promising. For a conventional LC grating, the theoretical diffraction efficiency of the first order beam is about 34\%. ${ }^{9}$ By contrast, a well-designed LC prism grating could reach $100 \%$ diffraction efficiency for the first order beam. ${ }^{7}$ Therefore, the LC-based prism grating is a strong contender for high efficiency laser beam steering. Several methods have been proposed to fabricate LC prism and prism grating. However, the fabrication methods and operation mechanisms of those deflectors are rather complicated. ${ }^{3,5-7}$ There is an urgent need to develop processes for LC prism grating.

Recently, we have demonstrated a prism grating using a periodic gradient refractive index nanoscale polymerdispersed liquid crystal (GRIN PDLC). ${ }^{10}$ Owing to the nanoscale LC droplets involved, the GRIN PDLC gratings are highly transparent in the whole visible region and independent of polarization. The shortcomings are in relatively high $\left(\sim 100 V_{\text {rms }}\right)$ operating voltage and small tunable phase change.

In this letter, we present a LC prism grating using polymer stabilized liquid crystal (PSLC) technique. The PSLC is produced by exposing the LC/monomer mixture with a uniform UV light through a periodically varying density filter. The formed PSLC structure exhibits a periodically gradient polymer networks. The LC in the regions stabilized by a higher polymer concentration usually exhibits a higher threshold voltage. ${ }^{11}$ Thus, when a homogeneous electric field is applied to the cell, the periodic gradient LC director distribution is generated and the prism effect occurs within each grating period. These microprisms help smooth the grating structure and boost the diffraction efficiency. In this approach, the LC concentration is around 95\% so that the operating voltage is relatively low.

Figure 1 illustrates the fabrication method of a PSLC prism grating. The key element is a patterned photomask. The photomask we used in this study is a periodically varying density pattern made by chromium with $300 \mu \mathrm{m}$ stripe width. When the uniform UV light (Loctite model 98016)

a)Electronic mail: swu@mail.ucf.edu passes through the photomask, the output intensity exhibits a saw-tooth profile within each period. The area with a stronger UV light would accelerate the polymerization process and aggregate a higher polymer concentration. Conversely, the area with a weaker UV exposure would have a lower polymer network concentration. As a result, a polymer network with periodic gradient morphology is formed. When a uniform electric field is applied, the LC directors are reoriented to different degrees. The region with a looser network packing has a lower threshold voltage than that of a denser region. Thus, the periodic gradient refractive index is formed within each grating period. As the applied voltage increases, the curvature of the gradient varies. In the high voltage regime, nearly all the LC molecules are aligned along the electric field direction. The gradient no longer exists and the grating effect vanishes.

In this experiment, nearly $3 \mathrm{wt} \% \mathrm{UV}$-curable monomer BAB-6 was added to LC host E48 (from Merck) along with a small amount of photoinitiator. The birefringence of E48 is $\Delta n=0.231$ at $\lambda=589 \mathrm{~nm}$ and room temperature. The BAB-6 monomer has a rod-like structure with a reactive double bond at both sides. The LC/monomer mixture was injected to an empty cell by capillary flow. The cell gap is $8 \mu \mathrm{m}$ and the thickness of the glass substrate is $1.1 \mathrm{~mm}$. The inner surfaces of the indium tin oxide glass substrates were overcoated with a thin polyimide layer and then rubbed in antiparallel directions. The curing UV intensity before the photomask is $12 \mathrm{~mW} / \mathrm{cm}^{2}$ and the exposure time is $60 \mathrm{~min}$.

In the voltage-off state, the prepared PSLC sample is highly transparent. A weak grating-like structure can still be observed by the naked eye. However, to resolve the detailed grating structures requires a polarizing optical microscope. On the microscope stage, the rubbing direction of the cell

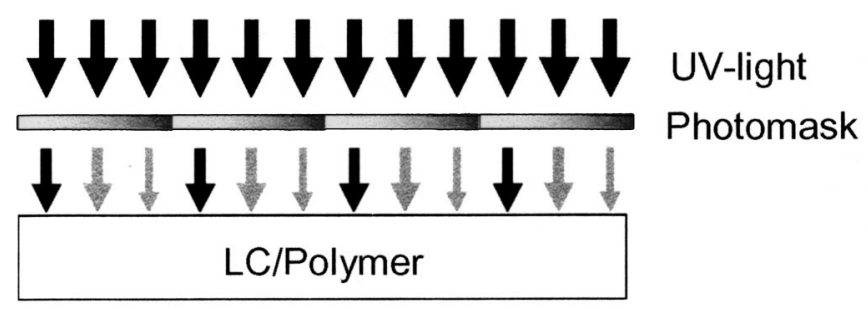

FIG. 1. Method for fabricating PSLC prism grating. The patterned photomask has periodic spatially varying grating structures. 


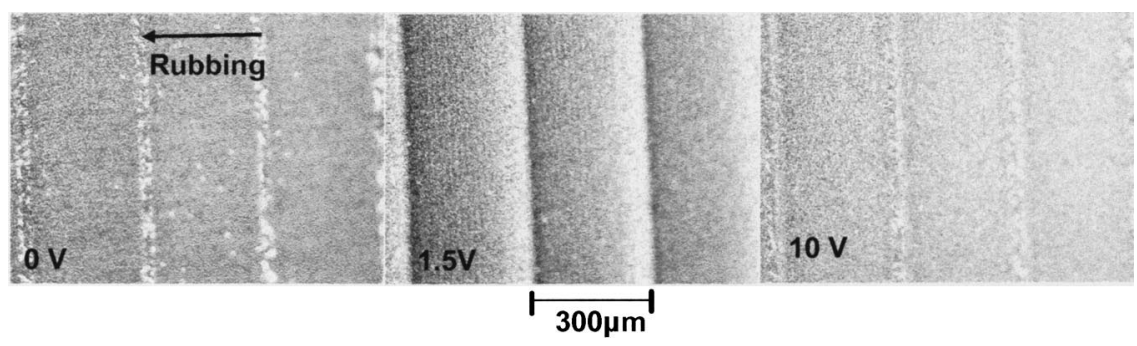

FIG. 2. Microscope photographs of the PSLC gratings taken at $V=0,1.5$, and $10 V_{\mathrm{rms}}$. The LC used is E48 and cell gap $d=8 \mu \mathrm{m}$.

was oriented at $45^{\circ}$ with respect to the fast axis of a linear polarizer and the analyzer was crossed to the polarizer. Three photographs of the grating structures were taken at $V=0$, 1.5, and $10 V_{\mathrm{rms}}$. Results are shown in Fig. 2.

From Fig. 2, the cell presents a weak grating-like structure at $V=0$. This is mainly due to the phase separation mechanism of the inhomogeneous PSLC, which is similar to that of the GRIN PDLC. ${ }^{10}$ The region having a looser polymer network has a higher LC concentration. As a result, the periodically inhomogeneous PSLC exhibits periodic gradient phase retardation within each grating period.

In our experiment we found that as the applied voltage increases, the LC directors within each grating period start to be reoriented from the right side and gradually extend to the left. This implies that the right border in each grating period has a lower threshold voltage than that of the left. As depicted in Fig. 1, the weaker UV exposure region leads to a lower polymer concentration which, in turn, results in a lower threshold voltage. This phenomenon also confirms that the gradient refractive index microprisms are indeed formed within each grating period. As the applied voltage reaches $1.5 V_{\text {rms }}$, the prism grating structure becomes clearer. However, at $10 V_{\text {rms }}$ the prism grating structure vanishes. This is because the LC directors are reoriented along the electric field direction so that the phase gradient is erased. From Fig. 2 , the borders of the periodical stripes are not very sharp. This is attributed to the smooth edges of the photomask patterns.

Figure 3 depicts the experimental setup for studying the diffraction characteristics of the PSLC prism grating. A linearly polarized $\mathrm{He}-\mathrm{Ne}$ laser beam $(\lambda=543.5 \mathrm{~nm})$ was used for this study. The laser polarization parallel to the cell rubbing direction was diffracted. We projected the diffraction patterns onto a screen. Figure 4 shows the three diffraction photographs taken at $V=0,1.5$, and $2 V_{\text {rms }}$. At $V=0$, the diffraction is asymmetric, i.e., most of the laser beam stays at the zeroth order and a small portion is diffracted to the first order. For an ideal prism grating, only positive diffraction

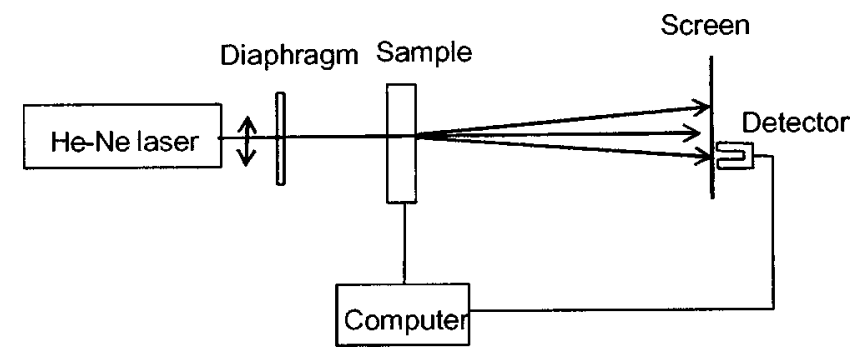

FIG. 3. The experimental setup used for studying the diffraction patterns and measuring the electro-optical properties of the PSLC prism grating. $\lambda$ $=543.5 \mathrm{~nm}$. orders should occur. However, in our PSLC grating a small amount of the laser beam is observed in the negative diffraction orders originating from the round prism structures shown in Fig. 2. At $V=1.5 V_{\text {rms }}$, most of the laser energy is diffracted to the first order. As the voltage is increased to $2 V_{\text {rms }}$, the zeroth order is almost vanished and most of the laser energy goes to the higher positive orders. Thus, our PSLC device has high first order diffraction efficiency while the zeroth order has a high extinguishing ratio. As compared to the stack crossed phase grating method for switching the zeroth order, ${ }^{12}$ our method is simpler.

To quantitatively characterize the diffraction properties of the PSLC grating, we measured the transmitted laser power through a pinhole. An ac voltage of $1 \mathrm{kHz}$ sinusoidal waves was applied to the PSLC grating through a computer controlled LabVIEW system. Figure 5 plots the voltagedependent transmittance of the zeroth and first orders. We use the following expression to quantify the relative zeroth and the first order diffraction efficiency of the PSLC prism grating

$$
\eta_{0,1}=T_{0,1} / T_{\text {total }} .
$$

In Eq. (1), $T_{0,1}$ denotes the transmitted power of the zeroth and the first order beam, respectively, and $T_{\text {total }}$ is the total transmitted laser power including all orders. For the PSLC grating we fabricated, we found $\eta_{1}<20 \%$ at $V=0$. As the applied voltage increases, $\eta_{1}$ increases. At $V=1.5 V_{\mathrm{rms}}, \eta_{1}$ reaches $80 \%$ efficiency. As the voltage increases to $2 V_{\text {rms }}$, $\eta_{0}$ is almost vanished, as shown in Fig. 4. At this stage, higher order diffractions occur so that $\eta_{1}$ is actually lower than $80 \%$. When the operating voltage exceeds $2 V_{\text {rms }}$, the prism effect of the diffraction grating is gradually weakening

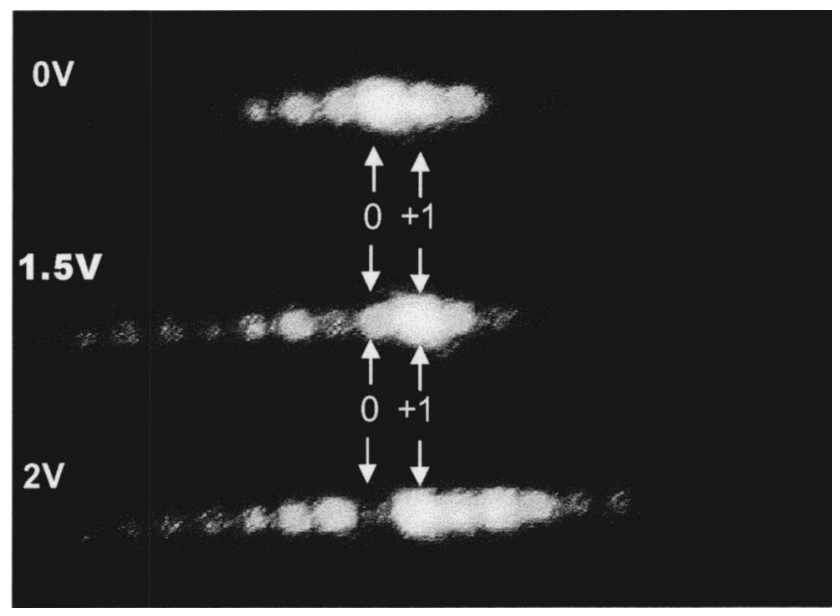

FIG. 4. Photographs of the diffraction patterns observed in a dark room at $V=0,1.5$, and $2 V_{\text {rms }}$. The PSLC prism grating has $300 \mu \mathrm{m}$ grating stripe. 


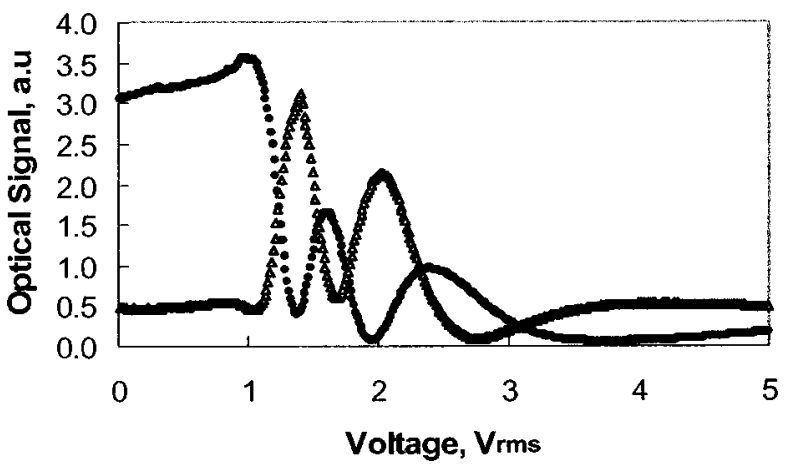

FIG. 5. Voltage-dependent relative transmittance of the zero (circles) and the first (triangles) order diffracted beams. The grating stripe width is 300 $\mu \mathrm{m}$.

and the prism grating behaves like a conventional LC grating. At a higher voltage $\left(>3 V_{\text {rms }}\right)$, light scattering takes place and the grating effect is degraded dramatically. The switching speed for the zeroth order was measured by using a square wave with $100 \mathrm{~ms}$ pulse width and $2 \mathrm{~V}$ amplitude. The turn-on time is $30 \mathrm{~ms}$ and turn-off time is $12 \mathrm{~ms}$.

Therefore, the PSLC prism grating can be operated in two regimes: $0-1.5 V_{\text {rms }}$ for high first order diffraction efficiency, or $0-2 V_{\text {rms }}$ for switchable zeroth order diffraction. At $V=0$, the $20 \%$ noise of the first order beam should be eliminated if an ideal phase grating is generated through a sharp photomask pattern. Under such a circumstance, the higher order diffractions should be also suppressed and the first order diffraction efficiency is likely to be boosted to $100 \%$ at $V=1.5 V_{\text {rms }}$.

Our grating period is $300 \mu \mathrm{m}$ which is a little too wide so that the diffraction angle is small. To solve this problem, one should use a photomask with narrow periodic stripe width. In the mean time, the thickness of the substrate near the photomask side should be as thin as possible in order to avoid the diffraction from the photomask during UV light exposure. To sharpen the borders of the PSLC prism grating stripes, it is necessary to use a photomask with sharp joint borders between the stripes. Our results only serve the purpose for proving concepts.

In conclusion, we have demonstrated an electrically switchable prism grating using polymer-stabilized nematic liquid crystal. The periodic gradient refractive index is realized by exposing the LC/monomer cell with UV light through a patterned photomask. The formed polymer network is a prism grating. That means the microprisms are formed within each grating period when a uniform electric field is applied across the LC cell. The optimized PSLC prism grating can be used as a beam deflector with high diffraction efficiency.

The authors would like to thank Yi-Pai Huang (National Chiao Tung University) for designing the photomask, and Dr. S. Gauza and Dr. Q. H. Wang for their technical assistance. This work is supported by DARPA under Contract No. DAAD19-02-1-0208.

${ }^{1}$ R. L. Sutherland, Appl. Phys. Lett. 64, 1074 (1994).

${ }^{2}$ W. M. Gibbons and S. T. Sun, Appl. Phys. Lett. 65, 2542 (1994).

${ }^{3}$ G. D. Love, J. V. Major, and A. Purvis, Opt. Lett. 19, 1170 (1994).

${ }^{4}$ J. Chen, P. J. Bos, H. Vithana, and D. L. Johnson, Appl. Phys. Lett. 67, 2588 (1995).

${ }^{5}$ D. P. Resler, D. S. Hobbs, R. C. Sharp, L. J. Friedman, and T. A. Dorschner, Opt. Lett. 21, 689 (1996).

${ }^{6}$ H. Okada, P. J. Bos, and H. Onnagawa, Jpn. J. Appl. Phys., Part 1 37, 2576 (1998).

${ }^{7}$ X. Wang, D. Wilson, R. Muller, P. Maker, and D. Psaltis, Appl. Opt. 39, $6545(2000)$.

${ }^{8}$ S. W. Kang, S. Sprunt, and L. C. Chien, Appl. Phys. Lett. 76, 3516 (2000).

${ }^{9}$ T. K. Gaylord and M. G. Moharam, Appl. Phys. B: Photophys. Laser Chem. 28, 1 (1982).

${ }^{10}$ H. Ren and S. T. Wu, Appl. Phys. Lett. 81, 3537 (2002).

${ }^{11}$ R. A. M. Hikmet and H. M. J. Boots, Phys. Rev. E 51, 5824 (1995).

${ }^{12}$ H. Sakata and M. Nishimura, Jpn. J. Appl. Phys., Part 1 39, 1516 (2000). 